PROCEEDINGS OF THE

AMERICAN MATHEMATICAL SOCIETY

Volume 140, Number 2, February 2012, Pages 415-428

S 0002-9939(2011)10925-0

Article electronically published on June 8, 2011

\title{
ARITHMETIC THEORY OF HARMONIC NUMBERS
}

\author{
ZHI-WEI SUN
}

(Communicated by Matthew A. Papanikolas)

\begin{abstract}
Harmonic numbers $H_{k}=\sum_{0<j \leqslant k} 1 / j(k=0,1,2, \ldots)$ play important roles in mathematics. In this paper we investigate their arithmetic properties and obtain various basic congruences. Let $p>3$ be a prime. We show that

$$
\sum_{k=1}^{p-1} \frac{H_{k}}{k 2^{k}} \equiv 0(\bmod p), \sum_{k=1}^{p-1} H_{k}^{2} \equiv 2 p-2\left(\bmod p^{2}\right), \sum_{k=1}^{p-1} H_{k}^{3} \equiv 6(\bmod p),
$$

and$$
\sum_{k=1}^{p-1} \frac{H_{k}^{2}}{k^{2}} \equiv 0(\bmod p) \quad \text { provided } p>5 .
$$

(In contrast, it is known that $\sum_{k=1}^{\infty} H_{k} /\left(k 2^{k}\right)=\pi^{2} / 12$ and $\sum_{k=1}^{\infty} H_{k}^{2} / k^{2}=$ $17 \pi^{4} / 360$.) Our tools include some sophisticated combinatorial identities and properties of Bernoulli numbers.

\section{INTRODUCTION}

The Riemann zeta function is given by

$$
\zeta(s)=\sum_{n=1}^{\infty} \frac{1}{n^{s}} \text { for } \operatorname{Re}(s)>1 .
$$

For $n \in \mathbb{N}=\{0,1,2, \ldots\}$ we define

$$
H_{n}=\sum_{0<k \leqslant n} \frac{1}{k}
$$

(Note that $H_{0}=0$ by convention.) These numbers are known as harmonic numbers, and they arise naturally from many fields of mathematics. For example, Euler found that

$$
\lim _{n \rightarrow+\infty}\left(H_{n}-\log n\right)=\gamma=0.577 \ldots \text { and } \sum_{n=1}^{\infty} \frac{H_{n}}{n^{2}}=2 \zeta(3) ;
$$

also,

$$
\sum_{n=1}^{\infty} \frac{H_{n}}{n 2^{n}}=\frac{\pi^{2}}{12} \quad \text { (S. W. Coffman [C], 1987) }
$$

Received by the editors July 22, 2010 and, in revised form, November 23, 2010.

2010 Mathematics Subject Classification. Primary 11B75; Secondary 05A19, 11A07, 11B68.

Key words and phrases. Harmonic numbers, congruences, Bernoulli numbers.

The author was supported by the National Natural Science Foundation (grant 10871087) of China.

(C)2011 American Mathematical Society Reverts to public domain 28 years from publication 
and

$$
\sum_{n=1}^{\infty} \frac{H_{n}^{2}}{n^{2}}=\frac{17}{360} \pi^{4} \quad \text { (D. Borwein and J. M. Borwein [BB], 1995). }
$$

For $m \in \mathbb{Z}^{+}=\{1,2,3, \ldots\}$, harmonic numbers of order $m$ are those rational numbers

$$
H_{n, m}=\sum_{0<k \leqslant n} \frac{1}{k^{m}} \quad(n=0,1,2, \ldots) .
$$

A famous result of Euler (cf. IR, pp. 231-232]) states that

$$
\lim _{n \rightarrow+\infty} H_{n, m}=\zeta(m)=(-1)^{m / 2-1} 2^{m-1} \pi^{m} \frac{B_{m}}{m !} \quad \text { for } m=2,4,6, \ldots,
$$

where $B_{0}, B_{1}, B_{2}, \ldots$, are Bernoulli numbers given by

$$
B_{0}=1 \text { and } \sum_{k=0}^{n}\left(\begin{array}{c}
n+1 \\
k
\end{array}\right) B_{k}=0(n=1,2,3, \ldots) .
$$

In this paper we focus on the arithmetic theory of harmonic numbers. In 1862 Wolstenholme [W] proved that if $p>3$ is a prime, then

$$
H_{p-1} \equiv 0\left(\bmod p^{2}\right) \text { and } H_{p-1,2} \equiv 0(\bmod p) ;
$$

hence

$$
\left(\begin{array}{c}
2 p-1 \\
p-1
\end{array}\right)=\prod_{k=1}^{p-1}\left(1+\frac{p}{k}\right) \equiv 1+p H_{p-1}+\frac{p^{2}}{2}\left(H_{p-1}^{2}-H_{p-1,2}\right) \equiv 1\left(\bmod p^{3}\right) .
$$

There are various extensions of the Wolstenholme theorem; see, e.g., A], ASVZ, $[\mathrm{B}, \mathrm{Gr}$, [HT] and $[\mathrm{Z}$. In particular, the paper $\mathrm{ASVZ}$ contains all Wolstenholmetype congruences of the form $\left(\begin{array}{l}a p+b \\ c p+d\end{array}\right) \equiv 1\left(\bmod p^{3}\right)$ with $a, b, c, d \in \mathbb{Z}$. In 1938 E. Lehmer $\left[\mathrm{L}\right.$ determined $H_{(p-1) / 2} \bmod p^{2}$ for any odd prime $p$. Though there are many identities and combinatorial interpretations involving harmonic numbers (see, e.g., $\overline{\mathrm{BPQ}}$ ), it seems that there are very few congruences for harmonic numbers.

In this paper we systematically investigate arithmetic properties of harmonic numbers and present some basic congruences for sums of terms involving harmonic numbers. Our main result is as follows.

Theorem 1.1. Let $p>3$ be a prime. Then

$$
\begin{aligned}
\sum_{k=1}^{p-1} \frac{H_{k}}{k 2^{k}} & \equiv 0(\bmod p), \\
\sum_{k=1}^{p-1} k^{2} H_{k}^{2} & \equiv-\frac{4}{9}(\bmod p), \\
\sum_{k=1}^{p-1} H_{k}^{3} & \equiv 6(\bmod p), \\
\sum_{k=1}^{p-1} H_{k}^{2} & \equiv 2 p-2\left(\bmod p^{2}\right) .
\end{aligned}
$$


When $p>5$ we have

$$
\sum_{k=1}^{p-1} \frac{H_{k}^{2}}{k^{2}} \equiv 0(\bmod p)
$$

Remark 1.1. Let $p>3$ be a prime. It is easy to determine $\sum_{k=1}^{p-1} k^{m} H_{k} \bmod p$ for all $m \in \mathbb{Z}$. (See $[\mathrm{ST}$, (5.4)] and its proof.) Note also that

$$
\begin{aligned}
\sum_{k=1}^{p-1} k H_{k} & =\sum_{k=1}^{p-1} k \sum_{j=1}^{k} \frac{1}{j}=\sum_{j=1}^{p-1} \frac{1}{j} \sum_{k=j}^{p-1} k=\sum_{j=1}^{p-1} \frac{1}{j}\left(\sum_{k=1}^{p-1} k-\sum_{i=0}^{j-1} i\right) \\
& =\frac{p(p-1)}{2} H_{p-1}-\sum_{j=1}^{p-1} \frac{j(j-1) / 2}{j} \equiv-\frac{(p-1)(p-2)}{4}\left(\bmod p^{3}\right) .
\end{aligned}
$$

Here are two consequences of Theorem 1.1.

Corollary 1.1. Let $p>3$ be a prime. Then

$$
\sum_{k=1}^{p-1} k H_{k}^{2} \equiv 1(\bmod p), \sum_{k=1}^{p-1} k H_{k}^{3} \equiv-3(\bmod p), \sum_{k=1}^{p-1} k^{3} H_{k}^{2} \equiv \frac{1}{6}(\bmod p) .
$$

Corollary 1.2. Let $p>3$ be a prime. Then

$$
\sum_{\substack{0 \leqslant r, s, t<p-1 \\ r+s+t \in\{p-1,2 p-2\}}} B_{r} B_{s} B_{t} \equiv 5(\bmod p) .
$$

Remark 1.2. Let $p>3$ be a prime. By taking $n=p-1$ in an identity of Matiyasevich (cf. [PS, (1.3)]), we get $\sum_{k=1}^{p-2} B_{k} B_{p-1-k} \equiv 1(\bmod p)$. Via a symmetric identity on Bernoulli polynomials given in [FPZ] (which is an extension of the main result of $[\mathrm{SP}]$ ), we may deduce that

$$
\sum_{\substack{0 \leq r, s, t<p-1 \\ r+s+t=p-1}} B_{r} B_{s} B_{t} \equiv \frac{5}{2}(\bmod p) .
$$

Our computation suggests the following refinement of (1.1) and (1.5).

Conjecture 1.1. For any prime $p>3$, we have

$$
\sum_{k=1}^{p-1} \frac{H_{k}}{k 2^{k}} \equiv \frac{7}{24} p B_{p-3}\left(\bmod p^{2}\right) \text { and } \sum_{k=1}^{p-1} \frac{H_{k}^{2}}{k^{2}} \equiv \frac{4}{5} p B_{p-5}\left(\bmod p^{2}\right) .
$$

For harmonic numbers of even order, we have the following conjecture based on our computation via Mathematica.

Conjecture 1.2. Let $n$ be any positive integer. If $p$ is a prime with $p-1 \nmid 6 n$, then

$$
\sum_{k=1}^{p-1} \frac{H_{k, 2 n}^{2}}{k^{2 n}} \equiv 0(\bmod p) .
$$

Furthermore, for each prime $p>6 n+1$ we have

$$
\sum_{k=1}^{p-1} \frac{H_{k, 2 n}^{2}}{k^{2 n}} \equiv \frac{s(n)}{6 n+1} p B_{p-1-6 n}\left(\bmod p^{2}\right),
$$


where

$$
s(n)=\frac{n}{2 n+1}\left(\begin{array}{c}
6 n+1 \\
2 n
\end{array}\right)+n .
$$

We make the following progress on Conjecture 1.2.

Theorem 1.2. Let $p>3$ be a prime and let $0<m<p-1$ be an even integer. Set $n=p-1-m$. Then

$$
\begin{aligned}
-m^{2} \sum_{k=1}^{p-1} \frac{H_{k, m}^{2}}{k^{m}} \equiv & \sum_{\substack{0 \leqslant i, j \leqslant n \\
i+j=3 n+2-(p-1)}}\left(\begin{array}{c}
n+1 \\
i
\end{array}\right)\left(\begin{array}{c}
n+1 \\
j
\end{array}\right) B_{i} B_{j} \\
& +\sum_{\substack{0 \leqslant i, j \leqslant n \\
i+j=3 n+2-2(p-1)}}\left(\begin{array}{c}
n+1 \\
i
\end{array}\right)\left(\begin{array}{c}
n+1 \\
j
\end{array}\right) B_{i} B_{j}(\bmod p) .
\end{aligned}
$$

Hence $\sum_{k=1}^{p-1} H_{k, m}^{2} / k^{m} \equiv 0(\bmod p)$ if $2 p / 3<m<p-1$.

In the next section we provide some lemmas. Section 3 is devoted to the proofs of Theorems 1.1-1.2 and Corollaries 1.1-1.2.

\section{Some Lemmas}

Lemma 2.1. Let $p$ be an odd prime. Then

$$
H_{p-k} \equiv H_{k-1}(\bmod p)
$$

and

$$
(-1)^{k}\left(\begin{array}{c}
p-1 \\
k
\end{array}\right) \equiv 1-p H_{k}+\frac{p^{2}}{2}\left(H_{k}^{2}-H_{k, 2}\right)\left(\bmod p^{3}\right)
$$

for every $k=1, \ldots, p-1$. If $p>3$, then

$$
\sum_{k=1}^{p-1} H_{k} \equiv 1-p\left(\bmod p^{3}\right) \text { and } \sum_{k=1}^{p-1} \frac{H_{k-1}}{k} \equiv 0(\bmod p) \text {. }
$$

Proof. When $k \in\{1, \ldots, p-1\}$, we clearly have

$$
H_{p-k}=\sum_{j=1}^{p-k} \frac{1}{j}=H_{p-1}-\sum_{j=1}^{k-1} \frac{1}{p-k+j} \equiv \sum_{j=1}^{k-1} \frac{1}{k-j}=H_{k-1}(\bmod p)
$$

and

$$
\begin{aligned}
& (-1)^{k}\left(\begin{array}{c}
p-1 \\
k
\end{array}\right)=\prod_{0<j \leqslant k}\left(1-\frac{p}{j}\right) \\
\equiv & 1-\sum_{0<j \leqslant k} \frac{p}{j}+\sum_{0<i<j \leqslant k} \frac{p^{2}}{i j} \\
\equiv & 1-p H_{k}+\frac{p^{2}}{2}\left(\left(\sum_{0<j \leqslant k} \frac{1}{j}\right)^{2}-\sum_{0<j \leqslant k} \frac{1}{j^{2}}\right)\left(\bmod p^{3}\right) .
\end{aligned}
$$

This proves (2.1) and (2.2). 
For the first congruence in (2.3) we observe that

$$
\begin{aligned}
\sum_{k=1}^{p-1} H_{k} & =\sum_{k=1}^{p-1} \sum_{j=1}^{k} \frac{1}{j}=\sum_{j=1}^{p-1} \frac{1}{j} \sum_{k=j}^{p-1} 1 \\
& =\sum_{j=1}^{p-1} \frac{p-j}{j}=p H_{p-1}-(p-1) \equiv 1-p\left(\bmod p^{3}\right)
\end{aligned}
$$

since $H_{p-1} \equiv 0\left(\bmod p^{2}\right)$.

Now we prove the second congruence in (2.3). Note that

$$
\sum_{k=1}^{p-1} \frac{p}{k}\left(\begin{array}{l}
p-1 \\
k-1
\end{array}\right)(-1)^{k}=\sum_{k=1}^{p-1}\left(\begin{array}{l}
p \\
k
\end{array}\right)(-1)^{k}+1+(-1)^{p}=(1-1)^{p}=0 .
$$

Thus,

$$
0=\sum_{k=1}^{p-1} \frac{(-1)^{k-1}}{k}\left(\begin{array}{l}
p-1 \\
k-1
\end{array}\right) \equiv \sum_{k=1}^{p-1} \frac{1-p H_{k-1}}{k} \equiv-p \sum_{k=1}^{p-1} \frac{H_{k-1}}{k}\left(\bmod p^{2}\right),
$$

and hence the desired congruence follows. We are done.

For a prime $p$ and an integer $a \not \equiv 0(\bmod p)$, as usual we call $q_{p}(a)=\left(a^{p-1}-1\right) / p$ a Fermat quotient.

Lemma $2.2[\mathrm{~L}$. Let $p>3$ be a prime. Then

$$
H_{(p-1) / 2} \equiv p q_{p}^{2}(2)-2 q_{p}(2)\left(\bmod p^{2}\right) .
$$

Lemma 2.3. Let $p>3$ be a prime. Then

$$
\sum_{\substack{k=1 \\ 2 \mid k}}^{p-1} \frac{H_{k}}{k} \equiv \frac{q_{p}^{2}(2)}{2}(\bmod p) \text { and } \sum_{\substack{k=1 \\ 2 \nmid k}}^{p-1} \frac{H_{k}}{k} \equiv-\frac{q_{p}^{2}(2)}{2}(\bmod p) .
$$

Proof. In view of (2.3),

$$
\sum_{k=1}^{p-1} \frac{H_{k}}{k}=\sum_{k=1}^{p-1} \frac{H_{k-1}}{k}+\sum_{k=1}^{p-1} \frac{1}{k^{2}} \equiv 0(\bmod p) .
$$

So it suffices to show the first congruence in (2.5).

For any odd $k \in\{1, \ldots, p-1\}$, clearly

$$
-\left(\begin{array}{c}
p-1 \\
k
\end{array}\right)=\left(\begin{array}{c}
p-1 \\
k
\end{array}\right)(-1)^{k} \equiv 1-p H_{k}\left(\bmod p^{2}\right)
$$

by (2.2). Thus, with the help of (2.4), we have

$$
\begin{aligned}
p \sum_{\substack{k=1 \\
2 \mid k}}^{p-1} \frac{H_{k-1}}{k} & \equiv \sum_{\substack{k=1 \\
2 \mid k}}^{p-1} \frac{1+\left(\begin{array}{c}
p-1 \\
k-1
\end{array}\right)}{k}=\frac{H_{(p-1) / 2}}{2}+\frac{1}{p}\left(\sum_{\substack{k=0 \\
2 \mid k}}^{p}\left(\begin{array}{l}
p \\
k
\end{array}\right)-1\right) \\
& \equiv \frac{p}{2} q_{p}^{2}(2)-q_{p}(2)+\frac{2^{p-1}-1}{p}=\frac{p}{2} q_{p}^{2}(2)\left(\bmod p^{2}\right),
\end{aligned}
$$


and hence

$$
\begin{aligned}
\sum_{\substack{k=1 \\
2 \mid k}}^{p-1} \frac{H_{k}}{k} & =\sum_{\substack{k=1 \\
2 \mid k}}^{p-1} \frac{H_{k-1}}{k}+\sum_{\substack{k=1 \\
2 \mid k}}^{p-1} \frac{1}{k^{2}} \\
& \equiv \frac{q_{p}^{2}(2)}{2}+\frac{1}{8} \sum_{k=1}^{(p-1) / 2}\left(\frac{1}{k^{2}}+\frac{1}{(p-k)^{2}}\right) \equiv \frac{q_{p}^{2}(2)}{2}(\bmod p),
\end{aligned}
$$

since $H_{p-1,2} \equiv 0(\bmod p)$. We are done.

Lemma 2.4. Let $n$ be any positive integer. Then

$$
\sum_{k=0}^{n-1} \frac{2^{k}}{k+1}=\sum_{\substack{k=1 \\
2 \nmid k}}^{n} \frac{1}{k}\left(\begin{array}{l}
n \\
k
\end{array}\right) .
$$

Proof. Observe that

$$
\begin{aligned}
\sum_{k=0}^{n-1} \frac{2^{k}}{k+1} & =\sum_{k=0}^{n-1} 2^{k} \int_{0}^{1} x^{k} d x=\int_{0}^{1} \sum_{k=0}^{n-1}(2 x)^{k} d x \\
& =\int_{0}^{1} \frac{(2 x)^{n}-1}{2 x-1} d x=\int_{0}^{1} \sum_{k=1}^{n}\left(\begin{array}{l}
n \\
k
\end{array}\right)(2 x-1)^{k-1} d x \\
& =\left.\sum_{k=1}^{n}\left(\begin{array}{l}
n \\
k
\end{array}\right) \frac{(2 x-1)^{k}}{2 k}\right|_{0} ^{1}=\sum_{k=1}^{n}\left(\begin{array}{l}
n \\
k
\end{array}\right) \frac{1-(-1)^{k}}{2 k} .
\end{aligned}
$$

Therefore, (2.6) follows.

Lemma 2.5. Let $n \in \mathbb{N}$. Then we have

$$
\sum_{k=0}^{n}\left(\begin{array}{l}
n \\
k
\end{array}\right)^{2}=\left(\begin{array}{c}
2 n \\
n
\end{array}\right) \text { and } \sum_{k=0}^{2 n}(-1)^{k}\left(\begin{array}{c}
2 n \\
k
\end{array}\right)^{3}=(-1)^{n} \frac{(3 n) !}{(n !)^{3}} .
$$

Proof. The first identity is a special case of the Chu-Vandermonde identity (cf. St, p. 12]). The second identity is due to Dixon (cf. [St, p. 45]).

Lemma 2.6. Let $p>3$ be a prime. Then

$$
\sum_{k=1}^{p-1} H_{k} H_{k, 2} \equiv 0(\bmod p) .
$$


Proof. Observe that

$$
\begin{aligned}
\sum_{k=1}^{p-1} H_{k} H_{k, 2} & =\sum_{k=1}^{p-1} H_{k} \sum_{j=1}^{k} \frac{1}{j^{2}}=\sum_{j=1}^{p-1} \frac{1}{j^{2}} \sum_{k=j}^{p-1} H_{k} \\
& =\sum_{j=1}^{p-1} \frac{1}{j^{2}}\left(\sum_{k=1}^{p-1} H_{k}-\sum_{0<s<j} H_{s}\right) \\
& =H_{p-1,2} \sum_{k=1}^{p-1} H_{k}-\sum_{j=1}^{p-1} \frac{1}{j^{2}} \sum_{0<s<j} \sum_{t=1}^{s} \frac{1}{t} \\
& \equiv-\sum_{j=1}^{p-1} \frac{1}{j^{2}} \sum_{0<t<j} \frac{1}{t} \sum_{t \leqslant s<j} 1=-\sum_{j=1}^{p-1} \frac{1}{j^{2}} \sum_{0<t<j} \frac{j-t}{t} \\
& =-\sum_{j=1}^{p-1} \frac{j H_{j-1}-j+1}{j^{2}} \equiv-\sum_{k=1}^{p-1} \frac{H_{k-1}}{k}(\bmod p) .
\end{aligned}
$$

Applying the last congruence in (2.3) we obtain (2.8) from the above.

Lemma 2.7. For $n \in \mathbb{Z}^{+}$we have the combinatorial identity

$$
\sum_{k=1}^{2 n-1} \frac{(-1)^{k-1}}{\left(\begin{array}{c}
2 n \\
k
\end{array}\right)} H_{k}=\frac{n}{2(n+1)^{2}}+\frac{H_{2 n}}{2 n+2} .
$$

Proof. This is a known identity which appeared as (2.18) of Gould [G, p. 20].

Let us recall some well-known properties of Bernoulli numbers (cf. IR, pp. 230238]). First,

$$
\sum_{j=0}^{k-1} j^{n}=\frac{1}{n+1} \sum_{j=0}^{n}\left(\begin{array}{c}
n+1 \\
j
\end{array}\right) B_{j} k^{n+1-j} \quad \text { for any } k, n \in \mathbb{Z}^{+} .
$$

Second, $B_{1}=-1 / 2$ and $B_{2 n+1}=0$ for $n=1,2,3, \ldots$ Third, if $p$ is an odd prime and $n$ is a positive integer not divisible by $p-1$, then $B_{n}$ is a $p$-adic integer (i.e., its denominator is not divisible by $p$ ) by the von Staudt-Clausen theorem.

Lemma $2.8[\mathrm{Z},(3.19)]$. Let $p>5$ be a prime. Then

$$
\sum_{k=0}^{p-3} B_{k} B_{p-3-k} \equiv 0(\bmod p) .
$$

Proof. Though (2.10) is known, here we provide a simple proof. Recall that $B_{0}, B_{1}$, $\ldots, B_{p-3}$ are $p$-adic integers. By an identity of Matiyasevich (cf. [PS, (1.3)] and $[\mathrm{SP}]$,

$$
(p-1) \sum_{k=2}^{p-5} B_{k} B_{p-3-k}-2 \sum_{k=2}^{p-5}\left(\begin{array}{c}
p-1 \\
k
\end{array}\right) B_{k} B_{p-3-k}=(p-2)(p-3) B_{p-3} .
$$

For $k \in\{2, \ldots, p-3\}$, we clearly have

$$
\left(\begin{array}{c}
p-1 \\
k
\end{array}\right) B_{k} \equiv(-1)^{k} B_{k}=B_{k}(\bmod p)
$$


Therefore, the identity yields

$$
\sum_{k=2}^{p-5} B_{k} B_{p-3-k} \equiv-2 B_{p-3}(\bmod p),
$$

which is equivalent to $(2.10)$ since $B_{p-4}=0$.

3. Proofs of Theorems 1.1-1.2 And Corollaries $1.1-1.2$

Proof of (1.1). Observe that

$$
\sum_{k=1}^{p-1} \frac{H_{k}}{k 2^{k}}=\sum_{k=1}^{p-1} \frac{H_{p-k}}{(p-k) 2^{p-k}} \equiv \sum_{k=1}^{p-1} \frac{2^{k-1} H_{k-1}}{-k}=-\sum_{k=0}^{p-2} \frac{2^{k} H_{k}}{k+1}(\bmod p),
$$

and hence

$$
p \sum_{k=1}^{p-1} \frac{H_{k}}{k 2^{k}} \equiv \sum_{k=0}^{p-1} \frac{2^{k}}{k+1}\left(\left(\begin{array}{c}
p-1 \\
k
\end{array}\right)(-1)^{k}-1\right)=\Sigma\left(\bmod p^{2}\right),
$$

where

$$
\begin{aligned}
\Sigma & :=\frac{1}{p} \sum_{k=0}^{p-2}\left(\begin{array}{c}
p \\
k+1
\end{array}\right)(-2)^{k}-\sum_{k=0}^{p-2} \frac{2^{k}}{k+1} \\
& =\frac{1}{-2 p} \sum_{j=1}^{p-1}\left(\begin{array}{c}
p \\
j
\end{array}\right)(-2)^{j}-\sum_{\substack{k=1 \\
2 \nmid k}}^{p-1} \frac{1}{k}\left(\begin{array}{c}
p-1 \\
k
\end{array}\right)(\text { by Lemma 2.4) } \\
& =\frac{(1-2)^{p}-1+2^{p}}{-2 p}+\sum_{\substack{k=1 \\
2 \nmid k}}^{p-1} \frac{\left(\begin{array}{c}
p-1 \\
k
\end{array}\right)(-1)^{k}}{k} .
\end{aligned}
$$

Thus

$$
\begin{aligned}
\Sigma+q_{p}(2) & \equiv \sum_{\substack{k=1 \\
2 \nmid k}}^{p-1} \frac{1-p H_{k}}{k}=H_{p-1}-\frac{H_{(p-1) / 2}}{2}-p \sum_{\substack{k=1 \\
2 \nmid k}}^{p-1} \frac{H_{k}}{k} \\
& \equiv-\frac{H_{(p-1) / 2}}{2}+\frac{p}{2} q_{p}^{2}(2) \equiv q_{p}(2)\left(\bmod p^{2}\right),
\end{aligned}
$$

in view of (2.4) and (2.5). We finally get

$$
p \sum_{k=1}^{p-1} \frac{H_{k}}{k 2^{k}} \equiv \Sigma \equiv 0\left(\bmod p^{2}\right)
$$

which yields (1.1).

Proof of (1.2). By Lemma 2.1,

$$
\sum_{k=0}^{p-1} k^{2} H_{k}^{2} \equiv \sum_{k=0}^{p-1} \frac{k^{2}}{p^{2}}\left(1-(-1)^{k}\left(\begin{array}{c}
p-1 \\
k
\end{array}\right)\right)^{2}(\bmod p) .
$$

Recall that

$$
\sum_{k=0}^{p-1} k^{2}=\frac{(p-1) p(2 p-1)}{6}
$$


Also,

$$
\begin{aligned}
& \sum_{k=0}^{p-1}(-1)^{k} k^{2}\left(\begin{array}{c}
p-1 \\
k
\end{array}\right)=\sum_{k=0}^{p-1}(-1)^{k}(k+k(k-1))\left(\begin{array}{c}
p-1 \\
k
\end{array}\right) \\
= & (p-1) \sum_{k=1}^{p-1}\left(\begin{array}{c}
p-2 \\
k-1
\end{array}\right)(-1)^{k}+(p-1)(p-2) \sum_{k=2}^{p-1}\left(\begin{array}{c}
p-3 \\
k-2
\end{array}\right)(-1)^{k-2} \\
= & (1-p)(1-1)^{p-2}+(p-1)(p-2)(1-1)^{p-3}=0 .
\end{aligned}
$$

Thus

$$
\begin{aligned}
p^{2} \sum_{k=0}^{p-1} k^{2} H_{k}^{2} & \equiv \frac{p(p-1)(2 p-1)}{6}+\sum_{k=0}^{p-1} k^{2}\left(\begin{array}{c}
p-1 \\
k
\end{array}\right)^{2} \\
& \equiv \frac{p}{6}(1-3 p)+\sum_{k=1}^{p-1}(p-1)^{2}\left(\begin{array}{c}
p-2 \\
k-1
\end{array}\right)^{2}\left(\bmod p^{3}\right) .
\end{aligned}
$$

With the help of the first identity in (2.7) and the Wolstenholme congruence,

$$
\begin{aligned}
\sum_{k=1}^{p-1}\left(\begin{array}{l}
p-2 \\
k-1
\end{array}\right)^{2} & =\sum_{j=0}^{p-2}\left(\begin{array}{c}
p-2 \\
j
\end{array}\right)^{2}=\left(\begin{array}{c}
2 p-4 \\
p-2
\end{array}\right)=\left(\begin{array}{c}
2 p-1 \\
p-1
\end{array}\right) \frac{p(p-1)}{2(2 p-1)(2 p-3)} \\
& \equiv \frac{p}{2} \cdot \frac{p-1}{3-8 p} \equiv \frac{p(p-1)}{18}(3+8 p) \equiv-\frac{p}{18}(5 p+3)\left(\bmod p^{3}\right) .
\end{aligned}
$$

Therefore

$$
p^{2} \sum_{k=0}^{p-1} k^{2} H_{k}^{2} \equiv \frac{p}{6}(1-3 p)-(p-1)^{2} \frac{p}{18}(5 p+3) \equiv-\frac{4}{9} p^{2}\left(\bmod p^{3}\right),
$$

and hence (1.2) follows.

Proof of (1.3). By Lemma 2.1,

$$
\sum_{k=0}^{p-1} H_{k}^{3} \equiv \sum_{k=0}^{p-1}\left(\frac{1-(-1)^{k}\left(\begin{array}{c}
p-1 \\
k
\end{array}\right)}{p}\right)^{3}=\frac{\Sigma}{p^{3}}(\bmod p),
$$

where

$$
\begin{aligned}
\Sigma & :=\sum_{k=0}^{p-1}\left(1-3(-1)^{k}\left(\begin{array}{c}
p-1 \\
k
\end{array}\right)+3\left(\begin{array}{c}
p-1 \\
k
\end{array}\right)^{2}-(-1)^{3 k}\left(\begin{array}{c}
p-1 \\
k
\end{array}\right)^{3}\right) \\
& =p-3(1-1)^{p-1}+3\left(\begin{array}{c}
2 p-2 \\
p-1
\end{array}\right)-(-1)^{n} \frac{(3 n) !}{(n !)^{3}} \quad \text { (by Lemma 2.5) }
\end{aligned}
$$

with $n=(p-1) / 2$.

Note that

$$
\left(\begin{array}{c}
2 p-2 \\
p-1
\end{array}\right)=\frac{p}{2 p-1}\left(\begin{array}{c}
2 p-1 \\
p-1
\end{array}\right) \equiv \frac{p}{2 p-1}\left(\bmod p^{4}\right)
$$


by the Wolstenholme congruence. Also,

$$
\begin{aligned}
(-1)^{n} \frac{(3 n) !}{(n !)^{3}} & =(-1)^{n} \frac{p}{p+n} \prod_{k=1}^{n} \frac{k(p-k)(p+k)}{k^{3}} \\
& =\frac{p}{p+n} \prod_{k=1}^{n}\left(1-\frac{p^{2}}{k^{2}}\right) \equiv \frac{p}{p+n}=\frac{2 p}{3 p-1}\left(\bmod p^{4}\right),
\end{aligned}
$$

since $2 \sum_{k=1}^{n} 1 / k^{2} \equiv H_{p-1,2} \equiv 0(\bmod p)$.

Combining the above, we obtain

$$
\sum_{k=0}^{p-1} H_{k}^{3} \equiv \frac{\Sigma}{p^{3}} \equiv \frac{p+3 p /(2 p-1)-2 p /(3 p-1)}{p^{3}} \equiv 6(\bmod p) .
$$

This proves (1.3).

Proof of (1.4). Taking $n=(p-1) / 2$ in (2.9) we get

$$
\sum_{k=1}^{p-2} \frac{(-1)^{k-1}}{\left(\begin{array}{c}
p-1 \\
k
\end{array}\right)} H_{k}=\frac{(p-1) / 2}{2(p+1)^{2} / 4}+\frac{H_{p-1}}{p+1},
$$

and hence

$$
\sum_{k=1}^{p-1} \frac{(-1)^{k-1}}{\left(\begin{array}{c}
p-1 \\
k
\end{array}\right)} H_{k} \equiv \frac{p-1}{(p+1)^{2}}\left(\bmod p^{3}\right) .
$$

By Lemma 2.1, for $k=1, \ldots, p-1$ we have

$$
\begin{aligned}
\frac{(-1)^{k}}{\left(\begin{array}{c}
p-1 \\
k
\end{array}\right)} & \equiv \frac{1-p^{3}\left(H_{k}-\frac{p}{2}\left(H_{k}^{2}-H_{k, 2}\right)\right)^{3}}{1-p\left(H_{k}-\frac{p}{2}\left(H_{k}^{2}-H_{k, 2}\right)\right)} \\
& \equiv 1+p\left(H_{k}-\frac{p}{2}\left(H_{k}^{2}-H_{k, 2}\right)\right)+p^{2}\left(H_{k}-\frac{p}{2}\left(H_{k}^{2}-H_{k, 2}\right)\right)^{2} \\
& \equiv 1+p H_{k}-\frac{p^{2}}{2}\left(H_{k}^{2}-H_{k, 2}\right)+p^{2} H_{k}^{2}\left(\bmod p^{3}\right) .
\end{aligned}
$$

Thus

$$
\frac{1-p}{(p+1)^{2}} \equiv \sum_{k=1}^{p-1} \frac{(-1)^{k}}{\left(\begin{array}{c}
p-1 \\
k
\end{array}\right)} H_{k} \equiv \sum_{k=1}^{p-1} H_{k}\left(1+p H_{k}+\frac{p^{2}}{2}\left(H_{k}^{2}+H_{k, 2}\right)\right)\left(\bmod p^{3}\right) .
$$

Note that

$$
\sum_{k=1}^{p-1} H_{k} \equiv 1-p\left(\bmod p^{3}\right) \text { and } \sum_{k=1}^{p-1} H_{k} H_{k, 2} \equiv 0(\bmod p)
$$

by (2.3) and (2.8). Therefore

$$
(1-p)\left(\frac{1}{(p+1)^{2}}-1\right) \equiv p \sum_{k=1}^{p-1} H_{k}^{2}+\frac{p^{2}}{2} \sum_{k=1}^{p-1} H_{k}^{3}\left(\bmod p^{3}\right)
$$

and hence (1.4) follows with the help of (1.3). 
Proof of (1.5). By Fermat's little theorem and the well-known formula for sums of powers,

$$
\begin{aligned}
\sum_{k=1}^{p-1} \frac{H_{k-1}^{2}}{k^{2}} & \equiv \sum_{k=1}^{p-1} \frac{\left(\sum_{j=0}^{k-1} j^{p-2}\right)^{2}}{k^{2}}=\sum_{k=1}^{p-1} \frac{1}{k^{2}}\left(\frac{1}{p-1} \sum_{j=0}^{p-2}\left(\begin{array}{c}
p-1 \\
j
\end{array}\right) B_{j} k^{p-1-j}\right)^{2} \\
& \equiv \sum_{k=1}^{p-1} \frac{1}{k^{2}}\left(\sum_{j=0}^{p-2}(-1)^{j} B_{j} k^{p-1-j}\right)\left(\sum_{i=0}^{p-2}(-1)^{p-2-i} B_{p-2-i} k^{i+1}\right) \\
& =-\sum_{k=1}^{p-1} \sum_{i, j=0}^{p-2}(-1)^{i+j} B_{j} B_{p-2-i} k^{p-2-j+i}(\bmod p) .
\end{aligned}
$$

It is well-known that for each $m \in \mathbb{Z}$ we have

$$
\sum_{k=1}^{p-1} k^{m} \equiv \begin{cases}-1(\bmod p) & \text { if } p-1 \mid m \\ 0(\bmod p) & \text { otherwise }\end{cases}
$$

(See, e.g., [IR, p. 235].) Note also that $B_{p-2}=0$. Thus

$$
\sum_{k=1}^{p-1} \frac{H_{k-1}^{2}}{k^{2}} \equiv \sum_{j=0}^{p-3}(-1)^{(j+1)+j} B_{j} B_{p-2-(j+1)}=-\sum_{j=0}^{p-3} B_{j} B_{p-3-j} \equiv 0(\bmod p)
$$

with the help of (2.10).

In view of (2.1) and the above,

$$
\sum_{k=1}^{p-1} \frac{H_{k}^{2}}{k^{2}}=\sum_{k=1}^{p-1} \frac{H_{p-k}^{2}}{(p-k)^{2}} \equiv \sum_{k=1}^{p-1} \frac{H_{k-1}^{2}}{k^{2}} \equiv 0(\bmod p) .
$$

This concludes the proof of (1.5).

Proof of Corollary 1.1. If $n \in \mathbb{Z}^{+}$, then

$$
\sum_{k=1}^{p-1} k H_{k}^{n}=\sum_{k=1}^{p-1}(p-k) H_{p-k}^{n} \equiv-\sum_{k=1}^{p} k H_{k-1}^{n}=-\sum_{j=1}^{p-1}(j+1) H_{j}^{n}(\bmod p),
$$

and hence

$$
\sum_{k=0}^{p-1} k H_{k}^{n} \equiv-\frac{1}{2} \sum_{k=1}^{p-1} H_{k}^{n}(\bmod p)
$$

Thus the first and the second congruences in Corollary 1.1 follow from (1.4) and (1.3) respectively. 
In view of $(1.2),(1.4)$ and the first congruence in Corollary 1.1,

$$
\begin{aligned}
\sum_{k=1}^{p-1} k^{3} H_{k}^{2} & =\sum_{k=1}^{p-1}(p-k)^{3} H_{p-k}^{2} \\
& \equiv-\sum_{k=1}^{p} k^{3} H_{k-1}^{2}=-\sum_{k=1}^{p-1}(k+1)^{3} H_{k}^{2} \\
& =-\sum_{k=1}^{p-1} k^{3} H_{k}^{2}-3 \sum_{k=1}^{p-1} k^{2} H_{k}^{2}-3 \sum_{k=1}^{p-1} k H_{k}^{2}-\sum_{k=1}^{p-1} H_{k}^{2} \\
& \equiv-\sum_{k=1}^{p-1} k^{3} H_{k}^{2}-3\left(-\frac{4}{9}\right)-3 \times 1-(-2)(\bmod p),
\end{aligned}
$$

and hence the third congruence in Corollary 1.1 also holds.

Proof of Corollary 1.2. Observe that

$$
\begin{aligned}
\sum_{k=1}^{p-1} H_{k}^{3} & =\sum_{k=1}^{p-1} H_{p-k}^{3} \equiv \sum_{k=1}^{p-1} H_{k-1}^{3} \equiv \sum_{k=1}^{p-1}\left(\sum_{j=0}^{k-1} j^{p-2}\right)^{3} \\
& \equiv \sum_{k=1}^{p-1}\left(\frac{1}{p-1} \sum_{j=0}^{p-2}\left(\begin{array}{c}
p-1 \\
j
\end{array}\right) B_{j} k^{p-1-j}\right)^{3} \\
& \equiv-\sum_{k=1}^{p-1}\left(\sum_{j=0}^{p-2}(-1)^{j} B_{j} k^{p-1-j}\right)^{3} \\
& =-\sum_{r, s, t=0}^{p-2}(-1)^{r+s+t} B_{r} B_{s} B_{t} \sum_{k=1}^{p-1} k^{3(p-1)-r-s-t} \\
& \equiv \sum_{\substack{0 \leqslant r, s, t<p-1 \\
r+s+t \in\{0, p-1,2 p-2\}}} B_{r} B_{s} B_{t}(\bmod p) .
\end{aligned}
$$

Therefore (1.6) follows from (1.3).

Proof of Theorem 1.2. For any $k \in\{1, \ldots, p-1\}$, obviously

$$
\begin{aligned}
H_{p-k, m} & \equiv \sum_{j=1}^{p-1} j^{p-1-m}-\sum_{0<j<k} \frac{1}{(p-k+j)^{m}} \\
& \equiv-\sum_{0<j<k} \frac{1}{(k-j)^{m}}=-H_{k-1, m}(\bmod p)
\end{aligned}
$$

since $p-1 \nmid m$ and $2 \mid m$. Thus

$$
\sum_{k=1}^{p-1} \frac{H_{k, m}^{2}}{k^{m}}=\sum_{k=1}^{p-1} \frac{H_{p-k, m}^{2}}{(p-k)^{m}} \equiv \sum_{k=1}^{p-1} \frac{H_{k-1, m}^{2}}{k^{m}}(\bmod p) .
$$


As $n=p-1-m$, we have

$$
\begin{aligned}
\sum_{k=1}^{p-1} \frac{H_{k-1, m}^{2}}{k^{m}} & \equiv \sum_{k=1}^{p-1} \frac{\left(\sum_{j=0}^{k-1} j^{n}\right)^{2}}{k^{m}}=\sum_{k=1}^{p-1} \frac{1}{k^{m}}\left(\frac{1}{n+1} \sum_{j=0}^{n}\left(\begin{array}{c}
n+1 \\
j
\end{array}\right) B_{j} k^{n+1-j}\right)^{2} \\
& \equiv \frac{1}{m^{2}} \sum_{k=1}^{p-1} k^{n}\left(\sum_{i=0}^{n}\left(\begin{array}{c}
n+1 \\
i
\end{array}\right) B_{i} k^{n+1-i}\right) \sum_{j=0}^{n}\left(\begin{array}{c}
n+1 \\
j
\end{array}\right) B_{j} k^{n+1-j} \\
& \equiv \frac{1}{m^{2}} \sum_{i, j=0}^{n}\left(\begin{array}{c}
n+1 \\
i
\end{array}\right)\left(\begin{array}{c}
n+1 \\
j
\end{array}\right) B_{i} B_{j} \sum_{k=1}^{p-1} k^{3 n+2-(i+j)}(\bmod p) .
\end{aligned}
$$

Note that $3 n+2<3(n+1)=3(p-m)<3(p-1)$. Hence (1.9) follows from the above.

If $2 p / 3<m<p-1$, then $3 n+2=3 p-1-3 m<p-1$. Hence the last two sums in (1.9) vanish, and consequently $\sum_{k=1}^{p-1} H_{k, m}^{2} / k^{m} \equiv 0(\bmod p)$.

The proof of Theorem 1.2 is now complete.

\section{ACKNOWLedgement}

The author would like to thank the referee for helpful comments.

\section{REFERENCES}

[A] E. Alkan, Variations on Wolstenholme's theorem, Amer. Math. Monthly 101 (1994), 1001-1004. MR1304326 (95g:11001)

[ASVZ] E. Alkan, J. Sneed, M. Vajaitu and A. Zaharescu, Wolstenholme matrices, Math. Rep. 58 (2006), 1-8. MR2268379 (2007f:11002)

[B] M. Bayat, A generalization of Wolstenholme's theorem, Amer. Math. Monthly 104 (1997), 557-560. MR.1453658 (98e:11007)

[BPQ] A. T. Benjamin, G. O. Preston and J. J. Quinn, A Stirling encounter with harmonic numbers, Math. Mag. 75 (2002), 95-103. MR1573592

[BB] D. Borwein and J. M. Borwein, On an intriguing integral and some series related to $\zeta(4)$, Proc. Amer. Math. Soc. 123 (1995), 1191-1198. MR.1231029 (95e:11137)

[C] S. W. Coffman, Problem 1240 and Solution: An infinite series with harmonic numbers, Math. Mag. 60 (1987), 118-119.

[FPZ] A. M. Fu, H. Pan and I. F. Zhang, Symmetric identities on Bernoulli polynomials, J. Number Theory 129 (2009), 2696-2701. MR2549525 (2010j:11039)

[G] H. W. Gould, Combinatorial Identities, Morgantown Printing and Binding Co., 1972. MR0354401 (50:6879)

[Gr] A. Granville, Arithmetic properties of binomial coefficients. I. Binomial coefficients modulo prime powers, in: Organic Mathematics (Burnaby, BC, 1995), pp. 253-276, CMS Conf. Proc., 20, Amer. Math. Soc., Providence, RI, 1997. MR1483922 (99h:11016)

[HT] C. Helou and G. Terjanian, On Wolstenholme's theorem and its converse, J. Number Theory 128 (2008), 475-499. MR.2389852 (2008k:11003)

[IR] K. Ireland and M. Rosen, A Classical Introduction to Modern Number Theory (Graduate Texts in Math., 84), 2nd ed., Springer, New York, 1990. MR.1070716 (92e:11001)

[L] E. Lehmer, On congruences involving Bernoulli numbers and the quotients of Fermat and Wilson, Ann. of Math. 39 (1938), 350-360. MR1503412

[PS] H. Pan and Z. W. Sun, New identities involving Bernoulli and Euler polynomials, J. Combin. Theory Ser. A 113 (2006), 156-175. MR2192774 (2006j:05020)

[St] R. P. Stanley, Enumerative Combinatorics, Vol. 1, Cambridge Univ. Press, Cambridge, 1999. MR1442260 (98a:05001)

[SP] Z. W. Sun and H. Pan, Identities concerning Bernoulli and Euler polynomials, Acta Arith. 125 (2006), 21-39. MR.2275215 (2007i:11037) 
[ST] Z. W. Sun and R. Tauraso, New congruences for central binomial coefficients, Adv. in Appl. Math. 45 (2010), 125-148. MR2628791

[W] J. Wolstenholme, On certain properties of prime numbers, Quart. J. Math. 5 (1862), $35-39$.

[Z] J. Zhao, Wolstenholme type theorem for multiple harmonic sums, Int. J. Number Theory 4 (2008), 73-106. MR2387917 (2008m:11006)

Department of Mathematics, Nanjing University, Nanjing 210093, People's Republic OF CHINA

$U R L:$ http://math.nju.edu.cn/ Zwsun

E-mail address: zwsun@nju.edu.cn 